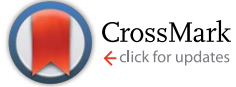

Cite this: RSC Adv., 2015, 5, 30701
Received 9th March 2015

Accepted 24th March 2015

DOI: $10.1039 / c 5 r a 04153 h$

www.rsc.org/advances

\section{Preparation of a sponge-like biocomposite agarose-chitosan scaffold with primary hepatocytes for establishing an in vitro 3D liver tissue model}

\begin{abstract}
Anuj Tripathi and Jose Savio Melo*
Designing a three-dimensional (3D) macroporous scaffold with desired bio-functional properties is an important aspect for fabricating an in vitro liver tissue model with applications in pre-clinical therapeutics testing. In the present study a bio-polymeric composite scaffold of agarose-chitosan (AG-CH) was synthesized at optimized sub-zero temperature and evaluated for its suitability in in vitro liver tissue engineering. The scaffold showed high porosity $(83 \pm 2 \%)$ with interconnected pores (average pore diameter 40-70 $\mu \mathrm{m})$. High swelling kinetics on account of the hydrophilic pore channels in the AG- $\mathrm{CH}$ scaffold allows unhindered migration of cells and gaseous exchange. At neutral $\mathrm{pH}$, the negative charge on the surface of the AG-CH scaffold ensures increased cell-to-cell interfacial interaction followed by colonization of hepatocytes. Rheological studies of the hydrated scaffold demonstrate its high spongelike visco-elastic behavior without any fracture deformation up to $34 \pm 1 \mathrm{~N}$, which insinuates its applicability for soft-tissue engineering. The AG-CH scaffold showed $\sim 15 \%$ degradation in a span of four weeks in sterile PBS at physiological $\mathrm{pH}$, which could help to maintain the structural integrity of neotissue formation. In vitro primary hepatocytes proliferation in the AG-CH scaffold showed an increase in cellular metabolic activity. The hepatic functions like albumin secretion and urea synthesis were established for the primary hepatocytes in the 3D scaffold and were higher in comparison to the control. The expression of hepatic CYP450 biomarker was observed in the in vitro cultured hepatocytes immobilized in the 3D AG-CH scaffold. Thus, the AG-CH scaffold with suitable physico-chemical properties and hepatic cell compatibility present its potential for developing an in vitro liver tissue model.
\end{abstract}

\section{Introduction}

The gold standard for evaluating the toxicological profile of therapeutic agents involves complex in vivo testing. Moreover, the use of animals, cost, time constraints and ever increasing number of therapeutics that need to be tested, are major concerns in the in vivo testing of drugs. Therefore, establishing in vitro $3 \mathrm{D}$ culture systems has become a priority for toxicologists. ${ }^{1}$ The use of in vitro tissue models has several advantages such as a decrease in animal numbers, the reduced cost for their maintenance, less time needed for drug testing, the requirement for a small quantity of chemicals and also an increase in throughput screening of multiple samples and their metabolites. ${ }^{\mathbf{1} 2}$ In the recent past, various in vitro liver tissue models have been developed using liver-derived components like liver tissue slices, immortalized liver cells, perfused liver and also primary hepatocytes. ${ }^{1}$ Among others, primary

Nuclear Agriculture and Biotechnology Division, Bhabha Atomic Research Centre, Mumbai - 400 085, India. E-mail: anujtri@barc.gov.in; jsmelo@barc.gov.in; Fax: +91-22-25505151; Tel: +91-22-25592760 hepatocytes and immortalized cell lines are most widely used in the in vitro liver tissue models. However, loss of cell viability and decrease in liver specific functions are the shortcoming of these models. Thus, recent developments are focused towards the in vitro engineering of $3 \mathrm{D}$ liver tissue and bio-artificial liver. ${ }^{3}$ Liver tissue engineering has emerged as a potential therapeutic approach to overcome these limitations for hepatotoxicity analysis and can replace or enhance the current standard practice of organ transplantation., ${ }^{3,4}$ A porous scaffold integrated with liver cells can recreate biological tissue substitutes with the structural and functional features of liver tissue.

Biomimetic scaffolds are a key component in 3D tissue engineering (TE) that balance cell adhesion and proliferation, temporary mechanical properties with amiable mass transport, to aid biological delivery and in vitro tissue regeneration. Several design processes were studied for the fabrication of $3 \mathrm{D}$ porous scaffolds, but were often found to be not adequate due to lack of control on scaffold architecture, porosity, and cellular interactions. ${ }^{5}$ More importantly, biocompatibility of the scaffold is imperative and must not elicit cytotoxic responses. The scaffold should have significant mechanical properties and should not 
disintegrate or deform during the application. ${ }^{6}$ Designing of a scaffold should also take into account the possibility for easy sterilization to prevent it from infection. ${ }^{7}$ A typical porosity of 80 to $90 \%$ with average pore diameter of at least $60-100 \mu \mathrm{m}$ is required for cell penetration and vascularization of the ingrown tissue. ${ }^{89}$ Afore mentioned requirements for scaffold material are numerous. To fulfill as many requirements as possible, several designing approaches have been proposed. ${ }^{\mathbf{1 0}}$ Today, foremost focus in designing of tissue engineering scaffolds is towards the use of natural polymers due to their inherent bioactive properties, which improve cellular interaction and proliferation. ${ }^{\mathbf{1 1}, 12}$ Interestingly, composites of natural polymers have shown increase in mechanical stability and improved cell interaction..$^{13-15}$ In a recent study, a hydrogel synthesized using de-cellularized liver ECM has also been proposed for liver tissue regeneration by encapsulating primary hepatocytes. ${ }^{\mathbf{1 6}}$

Among the several natural polymers, only a few like agarose and chitosan have the attractive properties required for liver tissue engineering. Agarose hydrogels have been used for seeding of hepatocytes and have shown enhanced production of extracellular matrix. ${ }^{\mathbf{1 7}}$ Agarose is also well documented for its high mechanical strength, controlled degradation and ability to maintain the cellular phenotype..$^{15,18,19}$ However, agarose alone is not suitable to mimic the microenvironment for hepatocytes. Thus, incorporation of ECM-like component could facilitate biomimetic properties in the porous 3D scaffold, chitosan obtained by the deacetylation of chitin, shows structural analogy to the important liver tissue component i.e. glycosaminoglycans (GAGs). ${ }^{20}$ Earlier studies reported that GAGs which is found in abundance in the extracellular matrix (ECM) of liver cells, induces synthesis and expression of gap junctions in the tissue and regulate intercellular communication in the liver. ${ }^{21}$ Chitosan has been shown to have excellent biocompatibility, biodegradability, non-toxicity, adsorption properties and ability to be degraded by lysozyme, a naturally occurring enzyme in human body fluid. ${ }^{22}$

In the present study, we have attempted to synthesize an elastic and degradable 3-D polymeric composite scaffold with interconnected macroporous architecture. The biopolymers agarose and chitosan were used in different concentrations to optimize the scaffold characteristics. The scaffold was characterized by physico-chemical and rheological techniques. Further, primary rat hepatocytes were grown under in vitro condition in the scaffold to examine the cell-material interaction by morphological analysis. Cell viability and functionality were accessed to evaluate the suitability of the scaffold for fabricating in vitro 3D liver tissue models.

\section{Experimental}

\subsection{Materials}

Chitosan (MW: 50 000-190 000 Da; degree of deacetylation $\geq 75 \%$ and viscosity: 20-300 cps), glutaraldehyde solution (25\%), sodium cyanoborohydride $\left(\mathrm{NaBH}_{3} \mathrm{CN}\right)$ (25\%), Dulbecco's modified Eagle's medium (DMEM), penicillin-streptomycin antibiotic, collagenase type I and 3-(4,5-dimethylthiazol-2-yl)2,5-diphenyltetrazolium bromide (MTT) ( $\geq 97.5 \%)$ were purchased from Sigma-Aldrich Chemie (Steinheim, Germany). Agarose (low EEO (-Mr): 0.09-0.13, gelling strength: $\sim 1200 \mathrm{~g} \mathrm{~cm}^{-2}$, gelling temperature: $\sim 38-40{ }^{\circ} \mathrm{C}$ ) was purchased from Sisco Research Laboratories (Mumbai, India). Dimethyl sulfoxide (DMSO) ( $\geq 99 \%$ ) was purchased from Merck (Mumbai, India). Glacial acetic acid was bought from $\mathrm{BDH}$ (Leicestershire, UK). Primary hepatocyte cells were gifted by Dr D. Singh (Yeungnam University, South Korea). All other chemicals were of analytical grade and used without further purification.

\subsection{Methods}

2.2.1. Preparation of agarose-chitosan (AG-CH) scaffolds. Chitosan powder (MW: 50 000-190 $000 \mathrm{Da}$; degree of deacetylation $\geq 75 \%$ and viscosity: $20-300 \mathrm{cps}$ ) was dissolved in $1 \%$ aqueous acetic acid solution $(\mathrm{pH} \sim 2.5)$ with the help of a mechanical stirrer at room temperature (final concentration; $0.5 \%, 1 \%$ and $1.5 \% ; \mathrm{w} / \mathrm{v})$. In separate $50 \mathrm{~mL}$ plastic tube, agarose (low EEO, -Mr: 0.09-0.13, gelling strength: $\sim 1200 \mathrm{~g}$ $\mathrm{cm}^{-2}$, gelling temperature: $\sim 38-40{ }^{\circ} \mathrm{C}$ ) (final concentration; $1 \%$, $2 \%, 3 \%, 4 \%$ and $5 \%$; w/v) was dissolved in deionized water $\left(\mathrm{dH}_{2} \mathrm{O}\right)$ by boiling until a clear transparent solution was obtained. Chitosan solution was then added to agarose solution in different polymer ratios (Table 1). These solutions were further cooled at room temperature till the solution temperature dropped to $\sim 45{ }^{\circ} \mathrm{C}$. Further, glutaraldehyde (80 $\mu \mathrm{L}$ of $25 \%$, $\mathrm{v} / \mathrm{v}$ dissolved in $420 \mu \mathrm{L}$ of $\mathrm{dH}_{2} \mathrm{O}$ ) was mixed gently to each polymer mixture, to a final concentration of $0.2 \%$ in $10 \mathrm{~mL}$ solution. Immediately the polymer solution was poured into moulds ( $2 \mathrm{~mL}$ and $5 \mathrm{~mL}$ plastic syringes) and incubated at $-12{ }^{\circ} \mathrm{C}$ for $16 \mathrm{~h}$ in a liquid cryo-bath. After the incubation, monoliths were thawed in $\mathrm{dH}_{2} \mathrm{O}$. Thereafter, the monoliths were extensively washed with $\mathrm{dH}_{2} \mathrm{O}$ followed by drying under vacuum at $-45{ }^{\circ} \mathrm{C}$ for $24 \mathrm{~h}$ (Martin Christ, Germany). These

Table 1 Optimization of polymers concentration for the synthesis of agarose-chitosan (AG-CH) scaffold ${ }^{a}$

Concentration of

polymers

\begin{tabular}{llll}
\hline AG & CH & Property of AG-CH scaffold \\
\hline $1 \%$ & $0.5 \%$ & & Gel not formed \\
& $1.0 \%$ & & Gel not formed \\
& $1.5 \%$ & & Brittle hydrogel \\
& $0.5 \%$ & & Weak hydrogel \\
& $1.0 \%$ & & Gel with uneven porosity \\
& $1.5 \%$ & & Brittle porous gel \\
$3 \%$ & $0.5 \%$ & Soft \& stable gel \\
& $1.0 \%$ & Porous, soft-spongy and stable \\
& $1.5 \%$ & Spongy but brittle \\
$4 \%$ & $0.5 \%$ & Spongy but non-elastic \\
& $1.0 \%$ & Porous, spongy \& stable \\
& $1.5 \%$ & Porous \& brittle \\
$5 \%$ & $0.5 \%$ & Decreased non-elasticity \& pores \\
& $1.0 \%$ & Increased stiffness \& decreased porosity \\
& $1.5 \%$ & Brittle \& decreased porosity
\end{tabular}

${ }^{a}$ Note: all the ratios of polymer were repeated five times. 
synthesized scaffolds were then stored in air-tight tubes at room temperature for further studies.

\subsubsection{Physico-chemical characterization of scaffolds}

A. Microstructure and porosity analysis. Surface gold coating of scaffolds were performed at $20 \mathrm{~mA}$ for $3 \mathrm{~min}$ using an ion sputter coater (Hoyeon Tech., Model-HC 21, South Korea) for studying the physical morphology of scaffolds using scanning electron microscope (SEM, FEI Quanta 200). The microscope was operated under high vacuum at $10 \mathrm{kV}$ with a sample spot size of 3 to $5 \mathrm{~mm}$. According to the Archimede's principle, porosity of scaffold was theoretically calculated using the following equation. ${ }^{23}$

$$
\text { Porosity }=\left(M_{\mathrm{W}}-M_{\mathrm{D}}\right) /\left(M_{\mathrm{W}}-M_{\mathrm{SUB}}\right)
$$

where, $M_{\mathrm{W}}$ is the water saturated wet mass of the scaffold, $M_{\mathrm{D}}$ is the dry mass of the scaffold, and $M_{\mathrm{SUB}}$ is the submerged mass of the scaffold.

B. Swelling kinetics and swelling ratio. The swelling analysis of AG-CH scaffold ( $8 \mathrm{~mm}$ diameter and $3 \mathrm{~mm}$ height) was carried out in phosphate buffer saline (PBS; pH 7.4) at room temperature $\left(27^{\circ} \mathrm{C} \pm 2\right)$ till the scaffold reached its equilibrium. The swelling kinetics (at regular time intervals) and swelling ratio (SR) were calculated using the following equations;

$$
\begin{gathered}
W_{\mathrm{U}}=\left[\left(W_{\mathrm{T}}-W_{\mathrm{D}}\right) / W_{\mathrm{E}}\right] \times 100 \\
\text { S.R. }=\left(W_{\mathrm{E}}-W_{\mathrm{D}}\right) / W_{\mathrm{D}}
\end{gathered}
$$

where, $W_{\mathrm{U}}$ is the water uptake capacity of the porous scaffold, S.R. is swelling ratio, $W_{\mathrm{T}}$ is the wet weight of the scaffold at different time intervals, $W_{\mathrm{D}}$ is the dry weight of the scaffold, and $W_{\mathrm{E}}$ is the wet weight of scaffold at swelling equilibrium.

C. Hydraulic permeability analysis. The hydraulic permeability of the polymeric monoliths was determined using Darcy's law which describes relationship between the liquid flow rate and pressure. The AG-CH scaffold ( $8 \mathrm{~mm}$ diameter, $5 \mathrm{~mm}$ height) was placed in the permeability measurement setup and a constant water pressure head was applied on the porous scaffold for $2 \mathrm{~min}$. The flushed water was then collected from the outlet and weighed. For the control experiment, no scaffold was placed between the flow-path. The hydraulic permeability was calculated by applying the recorded values to the following equation. ${ }^{23,24}$

$$
\kappa=\frac{\Delta X}{A \times M_{\mathrm{B} 2}} \times \frac{2 \pi^{2} r^{4}}{\left(M_{\mathrm{B} 1} / M_{\mathrm{B} 2}\right)^{2}-1}
$$

where, $\kappa$ is the hydraulic permeability of the porous scaffold, $A$ is the flushing area of the cylindrical scaffold, $\Delta X$ is the thickness of the scaffold, $M_{\mathrm{B} 1}$ and $M_{\mathrm{B} 2}$ are the mass of the flushed water from outlet in control setup and test setup, respectively.

D. In vitro degradation analysis. In vitro degradation of porous AG-CH scaffold was carried out by incubating the samples in sterile PBS solution ( $\mathrm{pH} 7.4)$ at $37{ }^{\circ} \mathrm{C}$ for 4 weeks under non-stirring condition. At weekly intervals, three samples were removed and dried at $60{ }^{\circ} \mathrm{C}$ in a hot air oven and then weighed. At the same time, PBS solution was replaced with fresh
PBS. The degree of degradation was calculated from the change in dry weight of scaffold

$$
\left.D_{\mathrm{D}}(\%)=\left(W_{\mathrm{I}}-W_{\mathrm{F}}\right) / W_{\mathrm{I}}\right) \times 100
$$

where, $D_{\mathrm{D}}$ is degree of degradation, $W_{\mathrm{I}}$ is initial dry weight of incubated sample and $W_{\mathrm{F}}$ is final dry weight of sample after incubation.

E. Zeta potential analysis. The sample preparation for zeta potential analysis of solids was carried out as per the earlier reported study. ${ }^{25}$ In brief, glutaraldehyde-crosslinked $\mathrm{AG}-\mathrm{CH}$ scaffold was dried ( $\sim 10 \mathrm{mg}$ dried mass) at $60{ }^{\circ} \mathrm{C}$ for $4 \mathrm{~h}$. The scaffold was then ground using mortar-pestle and suspended in $5 \mathrm{~mL}$ of ultra pure $\mathrm{H}_{2} \mathrm{O}$. The suspension was sonicated for $1 \mathrm{~h}$ at $100 \mathrm{mV}$ and $60 \%$ frequency and further analyzed using a Zetasizer analyzer (Nano-Z series, Malvern, UK). Before the analysis, the suspension was dispensed into different vials and pre-assigned $\mathrm{pH}$ values ( $\mathrm{pH} 4$ to 8 ) were adjusted with $1 \mathrm{~N} \mathrm{HCL}$ and $1 \mathrm{~N} \mathrm{NaOH}$ solutions without addition of background electrolytes.

F. Fourier transform infrared spectroscopy and thermogravimetricdifferential thermal analysis. The Fourier transform infrared (FT-IR) spectroscopy of native polymers and their composite forms were studied by FT/IR-660 Plus spectrophotometer (Jasco, Japan) at room temperature $(27 \pm 2)$. FT-IR samples were prepared in $\mathrm{KBr}$ in the ratio of $1: 10(\mathrm{w} / \mathrm{w})$, which were further analysed to understand the chemical attributions before and after polymer crosslinking. Thermogravimetric-differential thermal analysis (TG-DTA) (NETZSCH Thermal analyzer; STA 409 pc Luxx, GMBH) of the scaffold was examined from 0 to $700{ }^{\circ} \mathrm{C}$ at a heating increment of $10^{\circ} \mathrm{C} \min ^{-1}$ in an inert atmosphere.

G. Rheological characterization. The rheological characterization of AG-CH scaffold was performed on Rheometer-MCR 302 (Anton-Paar, Germany) using serrated plates of $8 \mathrm{~mm}$ diameter at $37{ }^{\circ} \mathrm{C}$. AG-CH scaffold ( $8 \mathrm{~mm}$ diameter and $3 \mathrm{~mm}$ height) was placed between the measuring plates and a gradually increasing force was applied from $1 \mathrm{~N}$ to $50 \mathrm{~N}$ at a fixed frequency $(1 \mathrm{~Hz})$ and amplitude $(0.1 \%)$. The associated software RHEOPLUS was used to record the different variables such as elastic modulus $\left(G^{\prime}\right)$, loss modulus $\left(G^{\prime \prime}\right)$ and shear stress $(\tau)$.

\subsubsection{Hepatocytes compatibility analysis}

A. Hepatocyte cell growth in $A G-C H$ scaffold. Hepatocytes were isolated from the liver of an adult male wistar rat (7-8 weeks, 200-215 g) by the liver perfusion method using $0.05 \%$ collagenase. The isolated hepatocytes were dispersed in a hormone-defined medium (HDM: Williams medium E supplemented with $10 \mu \mathrm{g} \mathrm{mL}^{-1}$ insulin, $0.1 \mu \mathrm{M} \mathrm{CuSO}_{4} \cdot 5 \mathrm{H}_{2} \mathrm{O}, 3 \mu \mathrm{g} \mathrm{mL} \mathrm{m}^{-1}$ $\mathrm{H}_{2} \mathrm{SeO}_{3}, 50 \mathrm{pM} \mathrm{ZnSO} \cdot 7 \mathrm{H}_{2} \mathrm{O}, 50 \mathrm{ng} \mathrm{mL}^{-1} \mathrm{EGF}, 50 \mu \mathrm{g} \mathrm{mL}^{-1}$ linoleic acid, $58.8 \mu \mathrm{g} \mathrm{mL} \mathrm{m}^{-1}$ penicillin and $100 \mu \mathrm{g} \mathrm{mL} \mathrm{mL}^{-1}$ streptomycin). These cells (cell density; $5 \times 10^{5}$ cells per $\mathrm{mL}$ ) were seeded drop wise onto medium-equilibrated AG-CH sections ( $5 \mathrm{~mm}$ height and $13 \mathrm{~mm}$ diameter) which were pre-sterilized by autoclaving. Cell seeded sections were then cultured in a humidified atmosphere of $5 \% \mathrm{CO}_{2}$ and $95 \%$ air at $37{ }^{\circ} \mathrm{C}$. The medium was gently replaced after $3 \mathrm{~h}$ of seeding and routinely after $24 \mathrm{~h}$ till end of the experiment. The cell-seeded sections on day 10 were fixed using $2.5 \%$ glutaraldehyde solution and then 
observed under SEM for cell-to-material interaction. Cell-viability was observed by inverted fluorescence microscope (Olympus, Japan) after fluorescien diacetate (FDA) staining. Cell proliferation in AG-CH scaffold was examined every alternate day upto day 10 using MTT assay. ${ }^{12,26}$ The relative cell viability (\%) was expressed as a percentage relative to the viable cells number.

B. Albumin and urea assay. For liver specific test, primary rat hepatocyte cells were seeded in the AG-CH scaffolds $(5 \mathrm{~mm}$ height and $13 \mathrm{~mm}$ diameter) placed in wells of 24-well tissue culture plates at a density of $1 \times 10^{5}$ cells per well. The medium was changed every other day. At pre-defined time intervals the culture medium was collected and stored at $-80{ }^{\circ} \mathrm{C}$ for analysis. In the medium, the secreted albumin was measured using enzyme-linked immunosorbent assay (ELISA Albumin Quantification Set; Bethyl, Montgomery, TX) and urea concentrations was determined using Urea assay kit (Sigma-Aldrich, Germany) according to the manufacturer's protocol.

C. Drug detoxification assay. Hepatic cytochrome $\mathrm{P} 450$ (CYP450) is a family of enzymes that catalyses the oxidative metabolism of drugs. It is expressed predominantly in the liver tissue and its induction is commonly used for identifying the fate of pharmaceutical residues. The CYP450 activity in the primary hepatocytes seeded into AG-CH scaffold was observed by incubating with freshly prepared $500 \mu \mathrm{L}$ of ethoxyresorufin (20 $\mu \mathrm{M}$ solution prepared in DMSO) (Sigma-Aldrich, Germany) on day 7. Non-fluorescent ethoxyresorufin metabolized into fluorescence resorufin by viable cells and thus generates fluorescent bright red color signals, which was monitored under fluorescence microscope.

2.2.4. Statistical analysis. For the statistical analysis, samples were studied in triplicate for all the experiments. The obtained experimental values are presented as mean \pm standard deviation (SD) for the material characterization. While, the data analysis of cell culture experiments was performed with one-way ANOVA using Tukey's honestly significant difference (HSD) test in SPSS software (version 10) and differences were considered significant at $P<0.05$.

\section{Results and discussion}

\subsection{Synthesis of AG-CH scaffold}

Agarose and chitosan polymer in the form of composite scaffold was synthesized under moderate freezing condition. At sub-zero temperature, phase separation occurs within the aqueous polymeric system, wherein, water separates from the polymers and forms ice crystals which act as a porogen. Simultaneously, polymer chains present in the unfrozen liquid micro-phase also get crosslinked. After a pre-defined time of incubation, an interconnected and crosslinked porous polymeric network was obtained due to void space formation by melting of the interconnected ice-crystals (porogen). For optimizing the scaffold properties, different ratios of agarose and chitosan were used in the study (Table 1). The optimum concentration of polymers ratio was obtained by setting up various preparations of different concentration of agarose and chitosan for achieving the elasticity and uniform porosity in the scaffold (Table 1). The optimum concentration of agarose to chitosan was found to be $3: 1$, respectively. In the control set, plain agarose and chitosan formed a 3-D porous scaffold, which was white and yellow in color, respectively.

However, the composite AG-CH scaffold was pale yellow in color (Fig. 1A). Plain agarose matrix displayed a soft tissue-like elastic property, while chitosan matrix was stiff and brittle in nature. In contrast, optimum concentration of both the polymers (total polymer concentration $4 \%$ ) provided a suitable elastic property to the composite scaffold (Fig. 1B-D). Beside bioactive property of chitosan, it is also suitable for providing stiffness to the scaffold at optimum concentration. In the composite, $1 \%$ chitosan was found to be suitable and above this concentration, scaffold displayed brittle nature. On the other hand, agarose provides the soft elastic property to the composite AG-CH scaffold. However, decreasing its concentration resulted in low matrix elasticity, while, increasing the concentration decreased the pore size in the scaffold. Moreover, plain agarose scaffolds showed poor reproducibility (30 $\pm 10 \%$ ) compare to composite AG-CH scaffolds (90 $\pm 10 \%$ ). Thus, $4 \%$ composite AG-CH scaffold comprising 3\% agarose and 1\% chitosan was used for further characterization.

\subsection{Morphology and porosity of AG-CH scaffolds}

Scanning electron microscope (SEM) was used for determining the porous architecture of scaffolds as well as orientation, randomness and size of pores within the 3D system. The horizontally cross-sectioned SEM images showed non-uniform distribution of pores in both the control scaffolds of agarose and chitosan. Large pore size (upto $\sim 250 \mu \mathrm{m}$ ) and random orientation were observed in agarose scaffolds. Besides, less randomness and small size of pores were seen in chitosan scaffold, however, the average length of pores was short with
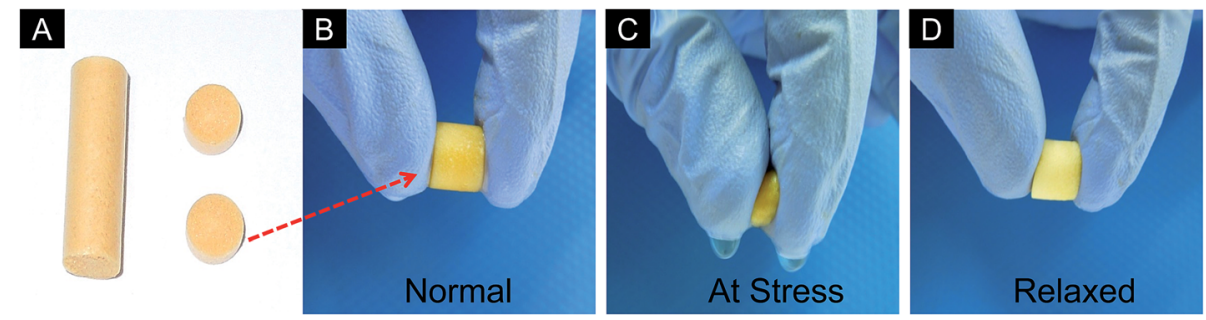

Fig. 1 Composite agarose-chitosan scaffold, (A) can be synthesize in various shapes like monolith and disc format, which shows sponge-like property in series of events; (B) normal (C) at stress and (D) relaxed. 

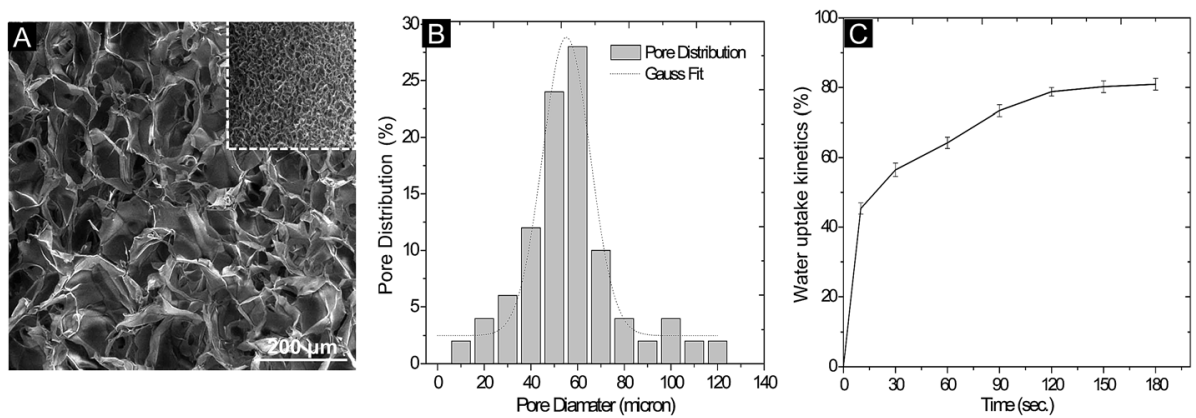

Fig. 2 Morphological characteristics of agarose-chitosan scaffold. (A) Scanning electron micrographs shows macroporous morphology and homogenous pore distribution, (B) which is having average pore size range of $40-70 \mu \mathrm{m}$ and (C) attain its equilibrium within 2 min due to interconnected pore network.

low surface area (data not shown), which is not appropriate for 3D cell growth. These aspects account for the fragile nature of chitosan scaffold. In contrast, AG-CH scaffold had a porous structure with homogenously distributed pores (Fig. 2A and inset) and showed interconnectivity and were more circular in shape. The pore diameter was found to be in the range of 10 to120 $\mu \mathrm{m}$, while the majority of pores were lying in between 40-70 $\mu \mathrm{m}$ (Fig. 2B). The distribution of pore wall thickness was in the range of 2 to $5 \mu \mathrm{m}$. The results suggest that the average pore diameter of the composite scaffold is large enough to facilitate the migration of mammalian cells.

\subsection{Swelling behavior of AG-CH scaffold}

A porous system consisting of interconnected pores allows the convective flow of liquid and gaseous exchange. Hence, a water uptake experiment was performed to examine the swelling as well as phase-transition behavior of AG-CH scaffold. The rate of water uptake by AG-CH scaffold with respect to time showed a $\geq 50 \%$ increase in weight by capacity within $30 \mathrm{~s}$ and it attained equilibrium within $2 \mathrm{~min}$ at room temperature $\left(27{ }^{\circ} \mathrm{C} \pm 2\right)$ (Fig. 2C). The swelling ratio of AG-CH scaffold was $15 \pm$ 0.25 (Table 2). Unlike classical hydrogel, a very slight and quick change in dimensions ( $\sim 1 \mathrm{~mm})$ was monitored during phasetransition from dry to wet. The high and quick water uptake capacity suggests that inter-pore-connections are present in the AG-CH scaffold, which could provide an efficient environment for 3-D cell adhesion and migration.

\subsection{Hydraulic permeability of AG-CH scaffold}

Growth of cells within the scaffold depends on the efficient supply of nutrient medium which should continuously permeate through the pores. The calculated average hydraulic permeability of the AG-CH scaffold was $4 \times 10^{-4} \mathrm{~m}^{4} \mathrm{~N}^{-1} \mathrm{~s}^{-1}$ (Table 2), which justifies the ability of macroporous AG-CH scaffold to efficiently permeate liquid through the interconnected pores unlike in the case of hydrogel synthesized at room temperature which contain mesopores. Earlier investigations on the design and characterization of scaffolds have revealed that molecular transport is significantly affected by pore interconnectivity and permeability. ${ }^{27}$ Therefore, beside high porosity, the interconnectivity and permeability can be viewed as an important property of the scaffold, which can be readily measured and related to its biological performance like controlled cell growth in a porous scaffold.

\subsection{Zeta potential of AG-CH scaffold}

The knowledge of electrokinetic charges present on the surface of scaffold could provide a cryptic nature of polymeric matrix which can assist the complex biological process. The electric potential between the interfacial regions near the matrix surface gives a broad idea about the surface chemistry of a material which is defined as its zeta potential or electro-osmotic mobility. Significant variation was observed in the zeta potential $(\zeta)$ of $\mathrm{AG}-\mathrm{CH}$ scaffold at different $\mathrm{pH}$ 's ranging from 4 to 8 (Fig. 3A). A range of $\mathrm{pH}$ was selected because in general, most of the cellular events occur in between this range. The AG-CH scaffold showed a negative zeta potential at neutral $\mathrm{pH}(-16.3 \pm$ $0.35 \mathrm{mV})$, which did not change significantly at pH $8(-18.0 \pm$ $1.4 \mathrm{mV}$ ). The point of zero charge (PZC) for AG-CH scaffold was observed at around $\mathrm{pH}$ 5.6. Below the $\mathrm{PZC}$, free amine groups $\left(\mathrm{R}-\mathrm{NH}_{2}\right)$ on chitosan are protonated in the ionic form $\left(\mathrm{R}^{-} \mathrm{NH}_{3}^{+}\right)$ and thus provide positive surface charge to the matrix. ${ }^{25}$ The negative zeta potentials above the PZC demonstrated possible surface interaction with $\mathrm{OH}^{-}$ions. In general, hydroxylated polymer surfaces exhibit a net negative charge at neutral $\mathrm{pH}$ which serves as a low affinity adhesion receptor by interacting to positively charged cell adhesion proteins that contains either hydrophobic amino acids or positively charged amino acids,

Table 2 Physico-chemical properties of AG-CH scaffold

Characteristics of agarose-chitosan (AG-CH) scaffold ${ }^{a}$

Porosity (\%)

Average pore diameter range $(\mu \mathrm{m})$

Swelling equilibrium (min)

Swelling ratio

Hydraulic permeability $\left(\mathrm{m}^{4} \mathrm{~N}^{-1} \mathrm{~s}^{-1}\right)$

Point of zero charge (PZC) at $\mathrm{pH}$

Degree of degradation (\%) (in four weeks)

$83 \pm 2$

40-70

$\sim 2$

$15 \pm 0.25$

$4 \times 10^{-4}$

$5.6 \pm 0.3$

$15 \pm 0.35$

${ }^{a}$ All data is represented as average of triplicates with standard deviation (SD). 
namely arginine and lysine.${ }^{28}$ Such interaction are vital in tissue engineering, as it is believed to act in tandem by binding to integrins and may be essential for the maintenance of normal cell phenotypes. ${ }^{29}$ Recent studies have also suggested that a net negative surface charge controls cell-to-cell and cell-to-material interaction in the 3-D porous scaffold. ${ }^{12}$ Considering the importance of cell-to-cell signaling in hepatocytes, low affinity surface of AG-CH scaffold holds potential as it can provide amiable cell-binding moieties to maintain the stable interaction of cell-to-matrix. Moreover, presence of chitosan which mimics natural GAGs like sequences can elicit biomimetic properties into the scaffold for functional cell growth.

\subsection{In vitro degradation of AG-CH scaffold}

Rate of degradation of AG-CH scaffold was examined under aseptic conditions in PBS at physiological $\mathrm{pH}(\mathrm{pH} 7.4)$ and temperature $\left(37^{\circ} \mathrm{C} \pm 1\right)$. The degradation was measured in terms of change in dry weight of the scaffold which was periodically recorded. AG-CH scaffold showed $15 \pm 0.35 \%$ degradation after four weeks of incubation in PBS. The scaffold showed a gradual degradation rate kinetic as shown in Fig. 3B, which can be attributed to the preferential hydrolytic scission of the hydrophilic polymeric network of agarose and chitosan. In general, long polymer chains (macromolecules) in the scaffold are degraded into small molecules (oligomers) which could disperse into the surrounding aqueous medium. ${ }^{30}$ Besides, compact interpenetrating network formed through crosslinking between the polymers can increase its chemical stability compared to non-crosslinked systems. ${ }^{31}$

\subsection{FTIR spectroscopy and thermal property of scaffold}

The presence of both polymers in the composite scaffold and conformational changes in their functional groups after crosslinking were examined by Fourier transform infrared (FTIR) spectroscopy (Fig. 3C). The spectrum of composite AG-CH scaffold represents a combination of functional groups present in the native polymer chains of agarose and chitosan. The characteristic peaks of 3,6-anhydro-L-galactose skeletal banding was observed at 931, 894 and $770 \mathrm{~cm}^{-1}$ in composite AG-CH scaffold, which confirms the presence of agarose. A broad band at $3200-3500 \mathrm{~cm}^{-1}$ is attributed to the $-\mathrm{NH}_{2}$ and $-\mathrm{OH}$ stretching vibrations. The absorption peaks at $1084 \mathrm{~cm}^{-1}$ confirms the presence of saccharide structure in composite scaffold. ${ }^{32}$ An absorption band at $1672 \mathrm{~cm}^{-1}$ is attributed to the formation of $\mathrm{C}=\mathrm{N}$ bond in the composite scaffold by glutaraldehyde crosslinking. ${ }^{25}$ Besides the corresponding characteristic absorption peaks, the symmetric and asymmetric stretching of $\mathrm{C}-\mathrm{H}$ at 2947 and $2891 \mathrm{~cm}^{-1}$ were observed. Peak at $1372 \mathrm{~cm}^{-1}$ represents the $\mathrm{C}-\mathrm{O}-\mathrm{C}$ stretching of ether. ${ }^{33}$ Few other similar peaks were found due to the same functional groups in the polymers and their composite. In addition, broadening of peaks were observed in composite AG-CH suggests the intermolecular complexation between polymer chains.

The thermal stability of polymer composite was confirmed by TG-DTA (Fig. 3D). TGA graph showed the first peak drop $(\sim 3 \%$ from initial weight of sample) at $100{ }^{\circ} \mathrm{C}$ due to loss of water molecules. The onset of scaffold degradation was observed at $208{ }^{\circ} \mathrm{C}$. Approximately $25 \%$ and $50 \%$ weight loss was observed at $268{ }^{\circ} \mathrm{C}$ and $318^{\circ} \mathrm{C}$, respectively. The lower rate of decomposition
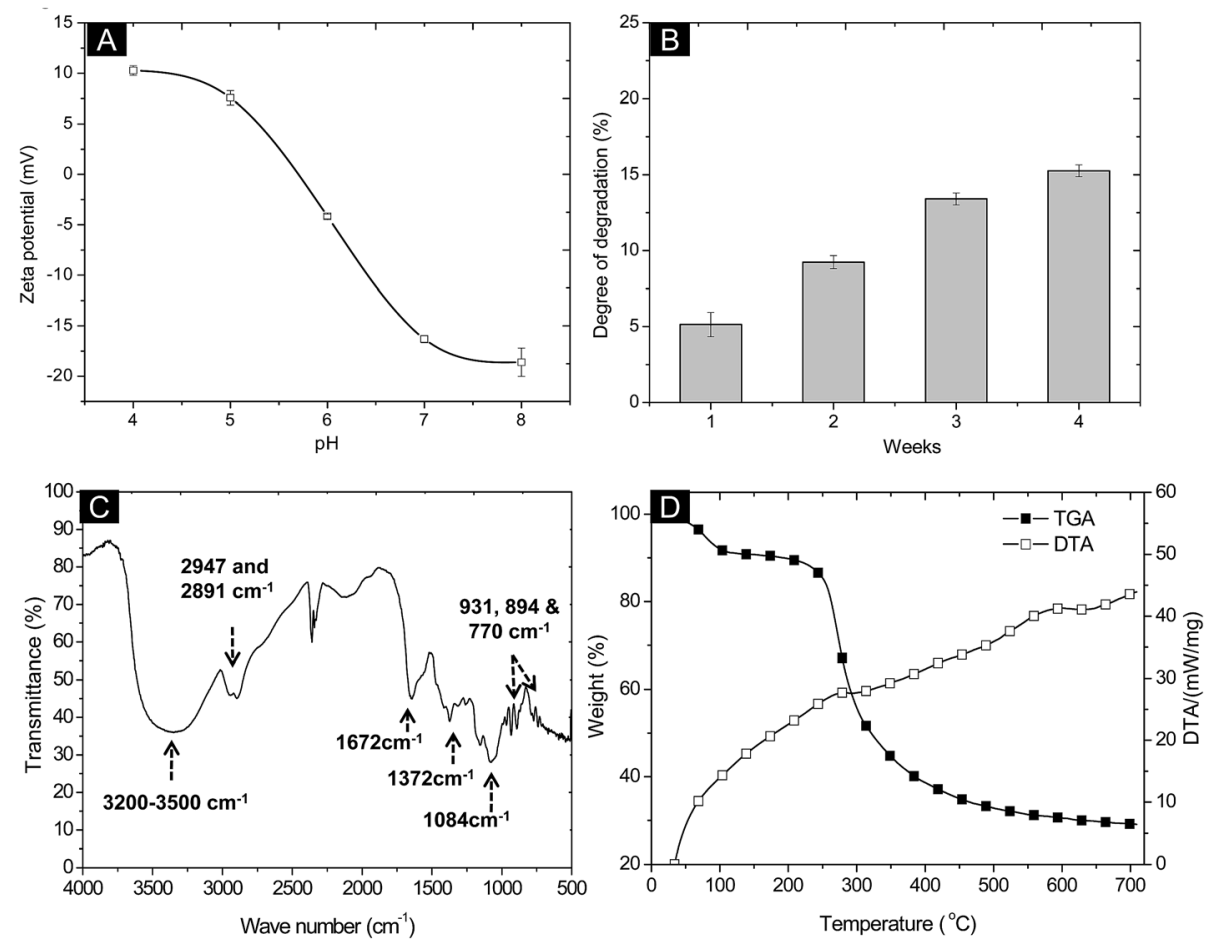

Fig. 3 Characterization of agarose-chitosan scaffold. (A) Surface charge analysis by zeta potential, (B) degree of degradation, (C) chemical group attribution by Fourier transform infrared spectroscopy and (D) thermal behaviour by TG-DTA analysis. 
is attributed to higher intermolecular bonding between polymers. The DTA graph showed two short exothermic peaks at $268{ }^{\circ} \mathrm{C}$ and $594{ }^{\circ} \mathrm{C}$, which is attributed to the possible physical (melting, vaporization and sublimation) and chemical (reduction and break-down) changes in the composite AG-CH scaffold. The solidus temperature of AG-CH scaffold was found to be $\sim 200{ }^{\circ} \mathrm{C}$. These results suggest that the scaffold can be autoclaved at $121{ }^{\circ} \mathrm{C}$ for sterile clinical applications and is in agreement with an earlier study on chitosan-based scaffold. ${ }^{13}$

\subsection{Rheological properties of AG-CH scaffold}

The polymeric scaffolds which are often soft materials can deform under applied force, leading to change in the stiffness (storage modulus; $G^{\prime}$ ) and fluidity (loss modulus; $G^{\prime \prime}$ ) of the scaffold. However, shear stress represents the fracture deformation limit of the material. The results were interpreted from the graph plotted between the storage modulus, loss modulus and shear stress against applied force from $1 \mathrm{~N}$ to $50 \mathrm{~N}$ at constant frequency $(1 \mathrm{~Hz})$ and amplitude $(0.1 \%)$ (Fig. 4). In the dry state, storage modulus $\left(G^{\prime}\right)$ of AG-CH scaffold was $2 \times 10^{6} \mathrm{~Pa}$ and did not show any significant change up to $6 \mathrm{~N}$, beyond which a significant change can be seen from the graph (Fig. 4A). The dynamic modulus of dried AG-CH scaffold was $6 \times 10^{6} \mathrm{~Pa}$. A deformation peak was observed at $27 \pm 1 \mathrm{~N}$. The loss modulus $\left(G^{\prime \prime}\right)$ of the scaffold showed similar pattern to $G^{\prime}$ and increasing values of $G^{\prime \prime}$ can be attributed to the loss of flow (viscous) property of the scaffold by increasing the force. In hydrated condition, a linear increment of $G^{\prime}$ and $G^{\prime \prime}$ seen in the graph (Fig. 4B) is suggestive of a resistance in the scaffold against applied force. The elastic modulus of hydrated AG-CH scaffold at $1 \mathrm{~N}$ was $1.6 \times$ $10^{5} \mathrm{~Pa}$ and dynamic modulus was $1 \times 10^{6} \mathrm{~Pa}$, which was significantly less compared to values under dry conditions. Unlike dry, the hydrated AG-CH scaffold did not show any retention time to maintain $G^{\prime}$ and $G^{\prime \prime}$, thus it indicates that the scaffold upon hydration loses its stiffness. Additionally, compared to dry scaffold, the hydrated scaffold showed delay in the fracture deformation i.e. $34 \pm 1 \mathrm{~N}$, which confirms the higher elastic property of the scaffold in their wet state. More often, non-uniform pore architecture in the scaffold dramatically diminishes the mechanical properties and restricts precise tissue procreation which is a vital concern in $3 \mathrm{D}$ cell culture systems. ${ }^{34}$ In contrast, a linear increment in the mechanical property of the AG-CH scaffold with respect to increasing force at constant frequency and amplitude provides an understanding of uniform distribution of pores resulting in a higher dynamic modulus (i.e. $1 \mathrm{MPa}$ to $6 \mathrm{MPa}$ ) with sponge-like property, indicating that macropores are interconnected in $\mathrm{AG}-\mathrm{CH}$ without compromising on its mechanical stability and thus holds promise for soft-tissue engineering.

\subsection{Hepatocytes compatibility and functionality analysis}

In order to show that the synthesized AG-CH scaffold has a potential in liver tissue engineering, an initial cell adhesion analysis was performed on $\mathrm{AG}-\mathrm{CH}$ scaffold using primary hepatocytes. At pre-defined time intervals, hepatocytes behavior in 3-D scaffold was examined by SEM and inverted fluorescence microscope, which showed viability and adherence of
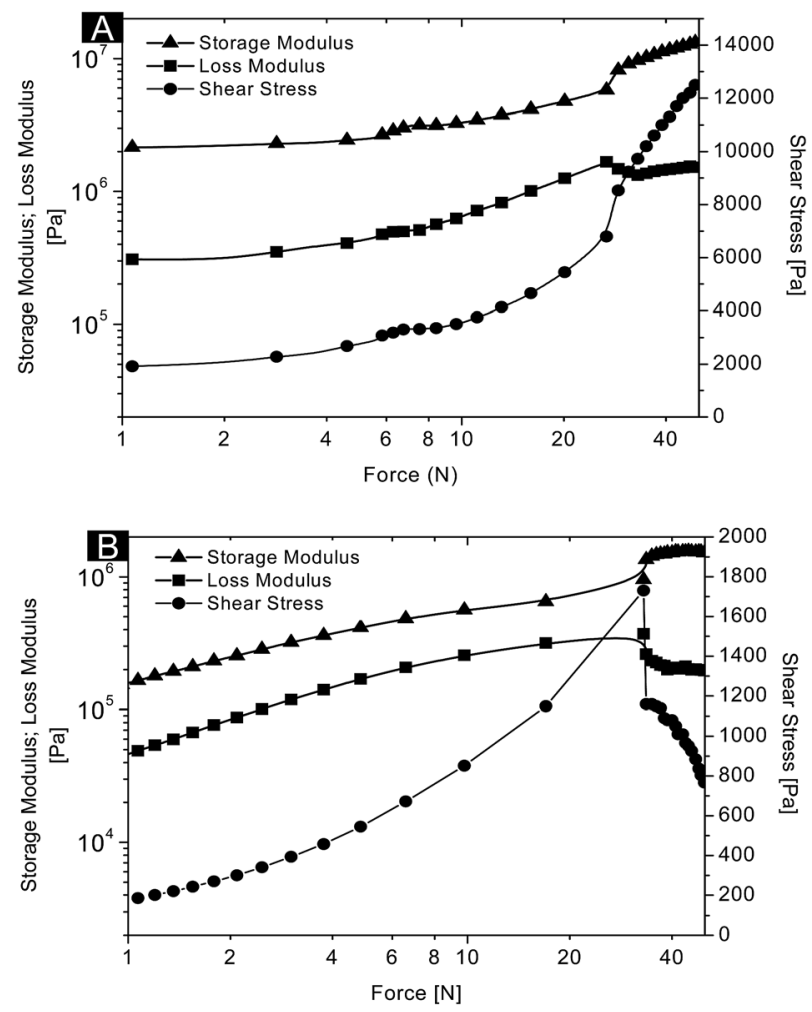

Fig. 4 Rheological behaviour of agarose-chitosan scaffold in its (A) dry and (B) wet conditions at $37^{\circ} \mathrm{C}$.

hepatocytes on the surface interface of AG-CH scaffold (Fig. 5). After day 10, SEM observations showed distribution of hepatocytes clusters throughout the surface of the AG-CH scaffold, wherein cells were retaining spherical morphology (Fig. 5A), which is a typical behavior of hepatocytes. Moreover, cell secreted extracellular matrix was also become visible after day 10 and delimited the adherence of hepatocytes. Apart from that, the FDA staining validates the viability and clustered cell growth of hepatocytes. These initially in vitro observations suggest that the scaffold was providing a native-like 3D micro-environment to the primary hepatocytes. Wherein, the pores of the scaffold were large enough to allow cells to migrate through the pores with effective delivery of nutrients as well as removal of metabolic waste which are essential for sustaining the cell functionality. The hepatocytes functionality and percent viability in AG-CH scaffold was monitored at periodic time intervals up to day 10 (Fig. 6A). The cell-compatibility of AG-CH scaffold was compared with the control (2D) cell culture $(0.1 \%$ gelatin-coated polystyrene tissue-culture plates). The calculated relative cell viability was found $\sim 60 \%$ after day 1 , which was decreased to $\sim 40 \%$ after day 10 in AG-CH scaffolds and showed less than one-fold decrease. In a control experiment, a significant threefold decrease in the cell viability from $\sim 45 \%$ to $\sim 15 \%$ was observed. However, percent cell viability was observed significantly higher in the AG-CH scaffold compared to the control over a period of in vitro cell culture. Unsuitable microenvironmental factors like limited surface area and 

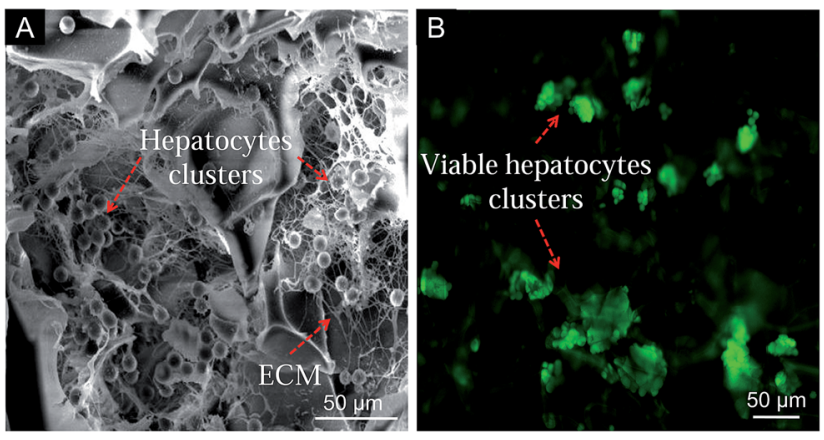

Fig. 5 Hepatocytes on agarose-chitosan scaffold after day 10. (A) SEM micrograph shows cell-to-material interaction, and (B) fluorescence FDA-staining shows hepatocytes viability.

insignificant cell-surface interaction in the control might be a reason of significant loss in hepatocytes viability.

Albumin secretion and urea synthesis by the growing hepatocytes were significantly higher in 3D scaffold compared to control (Fig. 6B and C). In comparison to control, AG-CH scaffolds showed approximate two-fold higher the albumin secretion and approximately three-fold higher the urea synthesis, after day 7. Correlating these liver specific functions with the cell viability suggest the possibility of initial physiological stresses to cells, followed by restoring of ingrown hepatocytes functions by adopting the 3-D microenvironment of AG-CH scaffold, which probably mimicking the liver-like microenvironment. Determination of CYP450 enzymatic activity in mammalian hepatocytes is a most commonly used method for screening of drug compound and detecting general toxicity, where CYP activity can be either induced or inhibited by specific compounds. The CYP450 activity of the seeded hepatocytes was monitored on day 7 , by the conversion of ethoxyresorufin to fluorescent resorufin. ${ }^{35}$ Since the biocatalytic conversion of ethoxyresorufin occurred inside hepatocytes, temporarily, the increase in fluorescent intensity of developed resorufin was higher within hepatocytes which can diffuse in the surrounding medium over a period of time. Therefore, sample was immediately observed under microscope after incubation. Fig. 7 shows high intensity of resorufin produced inside the cells that is depicting high expression of CYP450 and confers the hepatic functionality of growing hepatocytes. In addition, functional metabolic activity and cell-clusters of the primary hepatocytes were maintained during 3D culture which signifies that the nutrient transport and gaseous exchange in the AG-CH scaffold was quite efficient. These results are in agreement with the very recent study showing effective functionality of primary hepatocytes in a hydrogel made up of decellularized liver extracellular matrix (ECM) for liver tissue engineering. ${ }^{16}$ Unfortunately, these animal derived ECM for scaffold synthesis have shown limited biocompatibility and immunological concern. ${ }^{36}$ Moreover, ECM derived scaffolds are having a major concern of storage and immediate availability for clinical application.

Hepatocytes are very unstable and have tendency to lose their cell viability during the in vitro culture system. However,
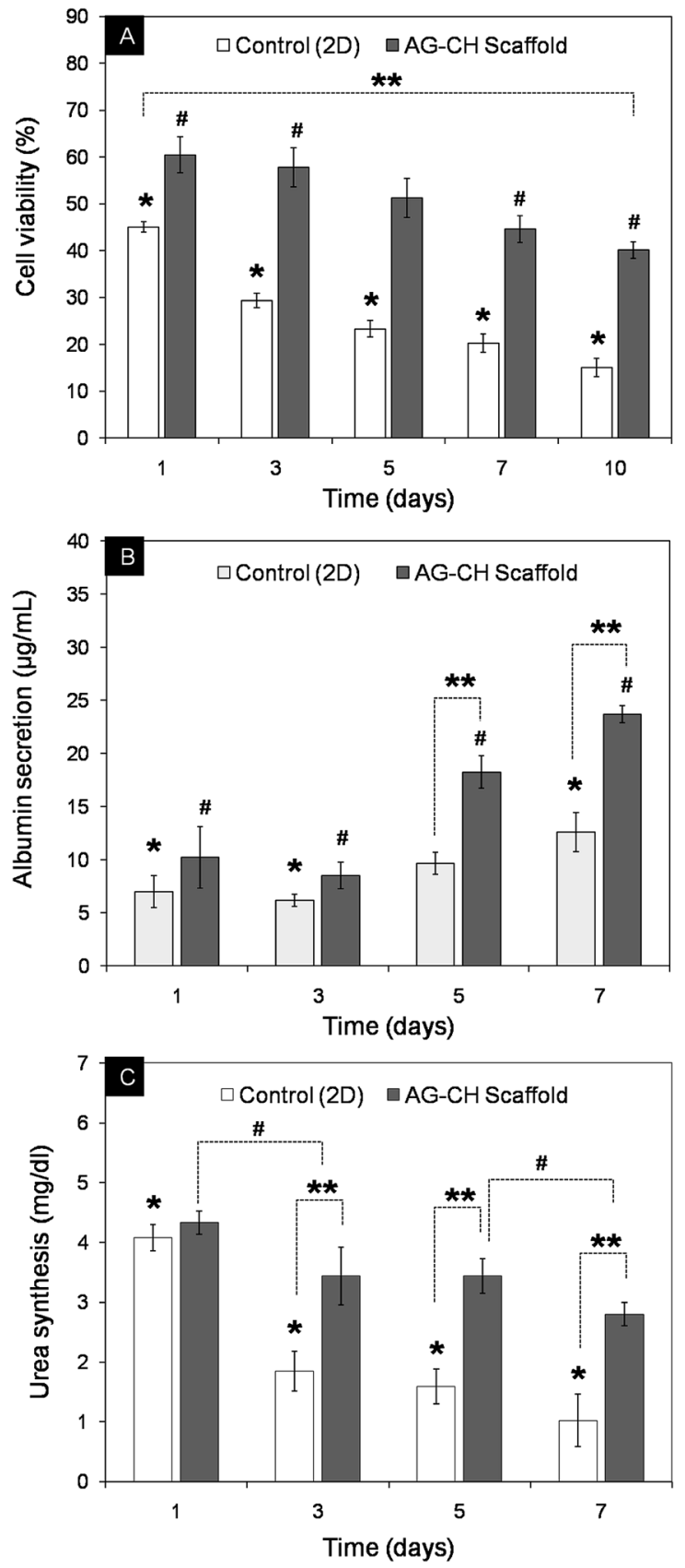

Fig. 6 In vitro quantification of cell functionality. (A) Percent cell viability, (B) albumin secretion and (C) urea synthesis of primary hepatocytes cultured in agarose-chitosan (AG-CH) scaffold. $(n=3$, $p<0.05$, * compared to control (2D); \# compared to AG-CH scaffold; ** compared 2D and AG-CH scaffold at each data point).

studies in rat and human hepatocytes have shown that when these cells were cultured under serum-free and hormonedefined medium (similar conditions were used in our study), hepatocytes maintain many markers of differentiation including cellular morphology (round and spheroid), express plasma proteins like albumin, and also activity of metabolic enzymes like CYP450. Functionally, these culture conditions also preserve hepatic stress response pathways. ${ }^{37}$ In agreement with these results, our finding show the expression of hepatic 


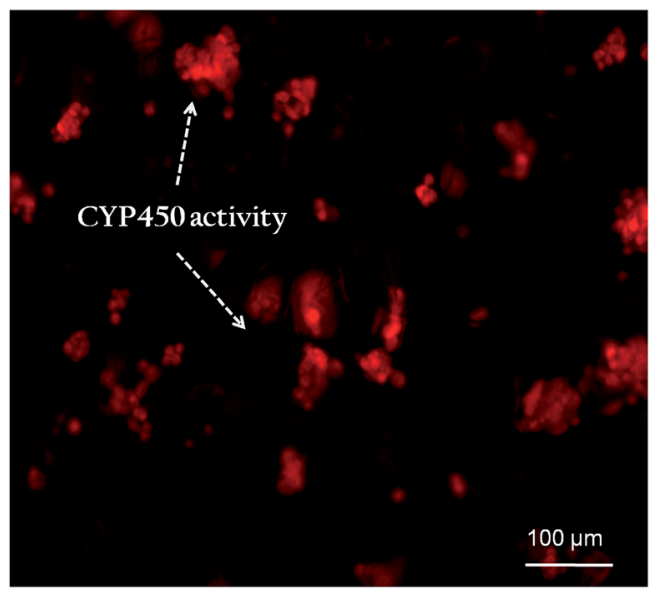

Fig. 7 Expression of CYP450 activity of rat primary hepatocytes immobilized in $\mathrm{AG}-\mathrm{CH}$ scaffold shows fluorescent red resorufin retained in the cell.

biomarker CYP450, maintain round morphology of cells and secret albumin during the in vitro 3D cell culture in AG-CH scaffold. Apart from that, in vitro micro-environment can influence the hepatic stress response which results in delayed functionality of hepatic cells in the 3D cell culture. This might be the reason for cells viability decrease irrespective of albumin secretion during the period of cell culture. There are several such reports that explain the similar cellular growth and functional profile of hepatocytes in the in vitro culture systems. ${ }^{16,38,39}$ Additionally, liver ECM not only allows anchorage of hepatocytes but also induce intracellular signaling pathways, thereby enabling sensing of the extracellular milieu with subsequent cellular adaptation to the environment. The complex 3D interaction is believed to be crucial in regulating and maintaining hepatic functions. Therefore, developments in the designing of synthetic scaffolds now offer a range of materials that can culture hepatocytes in 3D. Such artificial ECM as a scaffold for hepatocytes growth is an important key component in liver-like tissue modeling because the interactions of hepatocytes with the optimum artificial ECM and cell-cell contacts are essential in a $3 \mathrm{D}$ liver model to maintain hepatocyte polarization and functionality. ${ }^{40}$ Considering above parameters, our results are encouraging due to the high hepatocyte binding as well as high cell-cell interaction within interconnected pore network of AG-CH scaffold.

In the present study, the ratio of biocompatible and biodegradable natural polysaccharides like agarose and chitosan were optimized at cryo-conditions to achieve soft-tissue like elastic properties in the composite. There are only a few studies that have reported about the synthesis of composite matrices using agarose and chitosan, which were synthesized by different approaches and for different applications. For example, chitosan-agarose microspheres were synthesized by the water-in-oil (w/o) emulsion technique for the delivery of berbamine drug. ${ }^{41}$ Similarly, composite chitosan-agarose microgels were synthesized by the microfludic approach and shown for the delivery of anticancer drug i.e. 5-fluorouracil. ${ }^{42}$ In general, microfludic approach has the limitation of synthesizing gel with small pores and restriction on synthesis of large-size matrix like monolith scaffold. Also, a recent study has demonstrated synthesis of a conjugated chitosan-agarose hydrogel at room temperature for wound dressing application. ${ }^{43}$ In contrast, to the best of our knowledge, this is the first study which has shown the use of optimized concentration of agarose and chitosan to transform it into an elastic and sponge-like scaffold using cryo-polymerization process. The composite scaffold thus synthesized displayed fast hydration capacity due to interconnected pores which were uniformly distributed within the scaffold. Thus, it could quickly uptake the cells when re-hydrated with cell suspension, without using timeconsuming and expensive surface modulations with proteins or cell-adhesive peptides as in the case of classical hydrogels. By varying the processing method, the synthesis of spongy-like scaffold with optimum physical and biological properties, as well as with varying shapes (such as monolith, disc or membrane) can be tuned as per the requirement of size of tissue. These scaffolds also overcome the limitations of the classical hydrogels, such as reduced porosity, limited physical stability, poor sponginess, delicate handling due to high fragile nature and off-the-shelf unavailability. Unlike hydrogel, dried AG-CH scaffolds can be stored for years off-the-shelf at room temperature in a sterile environment and then it can be promptly utilized for studying drug metabolism and other liverspecific functions in a stable 3D in vitro system.

\section{Conclusions}

In conclusion, this study demonstrates the novel cryogenicsynthesis of AG-CH composite scaffold for fabricating in vitro $3 \mathrm{D}$ liver tissue models. We could achieve the optimal porosity of 40-70 microns with degradability and visco-elasticity properties. Scaffold showed interconnected macropores and biomimetic micro-environment which provides sufficient void volume for the growth and proliferation of primary hepatocytes and displayed liver cells functions under in vitro conditions. These features of scaffold will be valuable for constructing liverlike tissue model for analyzing the new therapeutic agent in the laboratory conditions and also could be optimized for bio-artificial liver development. Overall, results indicate that the sponge-like AG-CH scaffold has a potential in 3D liver tissue engineering. However, in vitro toxicological evaluation of these scaffolds will be the future direction for establishing pre-clinical application.

\section{Acknowledgements}

Authors would like to acknowledge Department of Atomic Energy, Government of India, for the financial assistance. Authors would like to acknowledge Dr D. Singh (Yeungnam University, South Korea) for gifting us primary hepatocyte cells. 


\section{Notes and references}

1 V. Y. Soldatow, E. L. LeCluyse, L. G. Griffith and I. Rusyn, Toxicol. Res., 2013, 2(1), 23-39.

2 E. F. A. Brandon, C. D. Raap, I. Meijerman, J. H. Beijnen and J. H. M. Schellens, Toxicol. Appl. Pharmacol., 2003, 189, 233246.

3 S. N. Bhatia, G. H. Underhill, K. S. Zaret and I. J. Fox, Sci. Transl. Med., 2014, 6, 245sr2.

4 J. S. Hammond, I. J. Beckingham and K. M. Shakesheff, Expert Rev. Med. Devices, 2006, 3, 21.

5 S. J. Hollister, Nat. Mater., 2005, 4, 518.

6 D. Singh, A. Tripathi, S. M. Zo, D. Singh and S. S. Han, Colloids Surf., B, 2014, 116, 502.

7 E. L. Chaikof, H. Matthew, J. Kohn, A. G. Mikos, G. D. Prestwich and C. M. Yip, Ann. N. Y. Acad. Sci., 2002, 961, 96.

8 S. Levenberg and R. Langer, Curr. Top. Dev. Biol., 2004, 61, 113.

9 V. Karageorgiou and D. Kaplan, Biomaterials, 2005, 26, 5474. 10 E. Sachlos and J. T. Czernuszka, Eur. Cells Mater., 2003, 5, 29. 11 A. Kumar and A. Tripathi, in Biotechnology in Biopolymers, ed. A. Tiwari and R. B. Shrivastava, i-Smithers Repra Publication Ltd., UK, ISBN-1847355439, 2012, ch. 9.

12 A. Tripathi, T. Vishnoi, D. Singh and A. Kumar, Macromol. Biosci., 2013, 13, 838.

13 N. Kathuria, A. Tripathi, K. K. Kar and A. Kumar, Acta Biomater., 2009, 5, 406.

14 H. Niiranen, T. Pyhalto, P. Rokkanen, M. Kellomaki and P. Tormala, J. Biomed. Mater. Res., Part A, 2004, 69, 699.

15 A. Tripathi, N. Kathuria and A. Kumar, J. Biomed. Mater. Res., Part A, 2009, 90, 680.

16 J. S. Lee, J. Shin, H. M. Park, Y. G. Kim, B. G. Kim, J. W. Oh and S. W. Cho, Biomacromolecules, 2014, 15, 206.

17 H. Ise, S. Takashima, M. Nagaoka, A. Ferdous and T. Akaike, Biotechnol. Lett., 1999, 21, 209.

18 K. W. Ng, C. C. Wang, R. L. Mauck, T. N. Kelly, N. O. Chahine, K. D. Costa, G. A. Ateshian and C. T. Hung, J. Orthop. Res., 2005, 23, 134.

19 P. D. Benya and J. D. Shaffer, Cell, 1982, 30, 215.

20 A. Kumar, A. Tripathi and S. Jain, J. Extra-Corpor. Technol., 2011, 43, 195.

21 D. C. Spray, M. Fujita, J. C. Saez, H. Choi, T. Watanabe, E. Hertzberg, L. C. Rosenberg and L. M. Reid, J. Cell Biol., 1987, 105, 541.
22 J. Hankiewicz and E. Swierczek, Clin. Chim. Acta, 1974, 57, 205.

23 A. Tripathi and A. Kumar, Macromol. Biosci., 2011, 11, 22.

24 J. Li and A. F. T. Mak, J. Biomater. Appl., 2005, 19, 253.

25 C. J. Luk, J. Yip, C. M. Yuen, C. Kan and K. Lam, J. Fiber Bioeng. Inf., 2014, 7, 35.

26 T. Mosmann, J. Immunol. Methods, 1983, 65, 55.

27 J. Fan, X. Jia, Y. Huang, B. M. Fu and Y. Fan, J. Tissue Eng. Regener. Med., 2013, DOI: 10.1002/term.1701, in press.

28 A. D. Cardin and H. J. Weintraub, Arteriosclerosis, 1989, 9, 21. 29 S. Saunders and M. Bernfield, J. Cell Biol., 1988, 106, 423.

30 A. Tripathi, A. B. Hadapad, R. S. Hire, J. S. Melo and S. F. D'Souza, Enzyme Microb. Technol., 2013, 53, 398.

31 M. Jurga, M. B. Dainiak, A. Sarnowska, A. Jablonska, A. Tripathi, F. M. Plieva, I. N. Savina, L. Strojek, H. Jungvid, A. Kumar, N. Firraz and C. McGuckin, Biomaterials, 2011, 32, 3423.

32 K. Prasad, G. Mehta, R. Meena and A. K. Siddhanta, J. Appl. Polym. Sci., 2006, 102, 3654.

33 Y. G. El-Reash, M. Otto, I. M. Kenawy and A. M. Ouf, Int. J. Biol. Macromol., 2011, 49, 513.

34 C. M. Murphy, M. G. Haugh and F. J. O'Brien, Biomaterials, 2010, 31, 461.

35 J. Fukuda and K. Nakazawa, Biomicrofluidics, 2011, 5(2), 022205.

36 U. Boeer, F. F. R. Buettner, M. Klingenberg, G. C. Antonopoulas, H. Meyer, A. Haverich and M. Wilhelmi, PLoS One, 2014, 9(8), e105964.

37 G. K. M. Olsavsky, E. M. Laurenzana and C. J. Omiecinski, Methods Mol. Biol., 2010, 640, 115-138.

38 S. Okada, Y. Kono and K. Shiraki, Yonago Acta Med., 1999, 42, 103-112.

39 R. H. Bhogal, J. S. Hodson, D. C. Bartlett, C. J. Weston, S. M. Curbishley, E. Haughton, K. T. Williams, G. M. Reynolds, P. N. Newsome, D. H. Adams and S. C. Afford, PLoS One, 2011, 6(3), e18222, DOI: 10.1371/ journal.pone.0018222.

40 P. Godoy, N. J. Hewitt, U. Albrecht and M. E. Andersen, et al., Arch. Toxicol., 2013, 87, 1315-1530.

41 H. U. Zhang, L. I. Sidong and Y. Lei, Polimeros, 2012, 22, 422. 42 V. Zamora-Mora, D. Velasco, R. Hernández, C. Mijangos and E. Kumacheva, Carbohydr. Polym., 2014, 111, 348.

43 S. P. Miguel, M. P. Ribeiro, H. Brancal, P. Coutinho and I. J. Correia, Carbohydr. Polym., 2014, 111, 366. 